\title{
COMPLEXITY OF THE FROG INTRACARDIAC NEURONS. INTRACELLULAR INJECTION STUDY
}

\author{
Darius Batulevicius ${ }^{1}$, Gertruda Skripkiene ${ }^{1}$, Vaida Batuleviciene ${ }^{2}$,

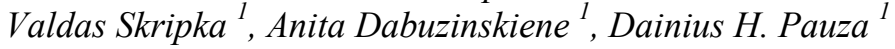 \\ ${ }^{1}$ Lithuanian University of Health Sciences, Medical Academy, \\ Institute of Anatomy, Lithuania \\ ${ }^{2}$ Kaunas College, Faculty of Health Care, Department of Social Health, Lithuania
}

\begin{abstract}
The goal of this study was to determine the structure of intracardiac neurons in the frog Rana temporaria. Fifty-six intracardiac neurons from 8 animals were labelled ionophoretically by the intracellular markers AlexaFluor 586 and Lucifer Yellow CH. Among the labelled neurons, we found the cells of unipolar, bipolar, multipolar and pseudounipolar types. Multiple neuronal processes originated from the soma, the axon hillock and the initial segment of axon. With respect to the soma, the neuron contained (Mean \pm SE) $3.5 \pm 0.3$ long and $5.5 \pm 0.6$ short processes. Most neurons had the spine, the bubble or the flake like extensions on their soma surface and were classified as Golgi I type neurons. Few Golgi II type neurons, the presumptive interneurons, were also found. Our findings contradict to a general view that the frog intracardiac ganglia contain only the adendritic neurons of the unipolar type. Our findings demonstrate that the frog intracardiac neurons are structurally complex and diverse. This diversity may account for the complicated integrative functions of the frog intrinsic cardiac ganglia.
\end{abstract}

Key words: heart, ganglion, cytology, nerve, dendrites, axon.

\section{INTRODUCTION}

Neuronal morphology and function are closely related, since the shape of the neuron determines its connections with other neurons and target tissues. The complexity of dendrites and the soma size correlates to the 
integrative capacity of the neuron [1]. It is considered that the neuron with complex dendrites receives more inputs than the adendritic neuron [1]. Likewise, the course of the axon may be indicative for the target specialization of the neuron. The neuron with a long axon extending to a distinct location is typically considered as a projecting neuron [2]. In contrast, the neuron with a short axon terminating proximal to the soma may be regarded as the interneuron or the local circuit neuron [2].

It has been shown recently that mammalian intracardiac ganglia contain functionally the diverse neurons including the parasympathetic, sympathetic, sensory and local circuit neurons [3-5]. Mammalian intracardiac neurons have variable morphologies, a different number of processes and multiple presynaptic inputs [3-6]. It is generally considered that the intracardiac neurons of amphibians exhibit surprising structural simplicity. The frog intracardiac ganglia contain only the adendritic neurons that receive a few presynaptic inputs and serve parasympathetic function [1]. It has been proposed that the frog intracardiac neurons could serve as a model to study the functional aspects of the intracardiac ganglia [1,7]. Therefore, there is a current need to review the knowledge on the structure of the frog intracardiac neurons.

In the present study we use the intracellular injection technique to determine the structure of the frog intracardiac neurons. We report that the frog intracardiac neurons exhibit remarkable morphological diversity with respect to the soma and neuronal processes.

\section{MATERIAL AND METHODS}

The study was performed on 8 adult (20-35 g in weight) common frogs (Rana temporaria). This investigation conforms both to the "Principles of laboratory animal care" (NIH publication No 86-23, revised 1985) and to the local guidelines for the use of experimental animals. Frogs were euthanized by the ether overdose.

\section{Intracellular injection of neurons}

The atria with interatrial septum, venal sinus and atrio-ventricular region were dissected and pinned flat in the Petri dish with the physiologic solution ( $\mathrm{pH} 7,2$; composition in $\mathrm{mM}$ : $\mathrm{NaCl}, 114 ; \mathrm{KCl}, 4,8 ; \mathrm{CaCl}_{2}, 1,8$; $\left.\mathrm{MgCl}_{2}, 1,44\right)$. The neuron somata were observed by a phase contrast 
illumination on the microscope Axiovert $40 \mathrm{CFL}$ (Zeiss). Intracellular fluorescent dyes Lucifer Yellow $\mathrm{CH}$ (Aldrich) and AlexaFluor 586 (Molecular Probes) were injected ionophoretically into the somata of neurons. The microelectrodes were made from borosilicate glass capillaries $(0.86 \mathrm{~mm}$ inner diameter, Intrafil) with puller PC-10 (Narishige). The microelectrode was inserted into the neuron soma by the aid of micromanipulator ROE-200 (Sutter Instrument Company). The dyes were injected by applying $10 \mathrm{nA}$ constant negative current for 10-15 min with Microionophoresis Dual Current Generator 260 (World Precision Instruments).

\section{Microscopy of the injected neurons}

Following the injection, the tissue was fixed in 4\% paraformaldehyde in PBS overnight. Then the tissue was washed in PBS for $30 \mathrm{~min}$ and mounted on glass slides using the Vectashield mounting medium (Vectorlabs). The neurons were observed at $400 \mathrm{X}$ magnification by the fluorescent microscope Imager Z1 (Zeiss) equipped with an Apotome (Zeiss) and digital camera AxioCam MRm (Zeiss). We used the fluorescein isothiocyanate (FITC) and cyanine (Cy3) fluorescence filters to observe Lucifer Yellow $\mathrm{CH}$ and AlexaFluor586 labelled neurons, respectively. Measurements and reconstructions of neurons were made with AxioVision 4.7 image analysing software (Zeiss).

\section{RESULTS}

\section{Neuron soma}

Round, oval and ovoid shaped neurons predominated in the frog heart (Figure 1). In addition, we found spindle, crescent and triangular shaped neuron somata. The long axis of the labelled soma was $40 \pm 1 \mu \mathrm{m}$, while the short axis $29 \pm 1 \mu \mathrm{m}$ (Mean $\pm \mathrm{SE}$ ). The soma occupied $1004 \pm$ $61 \mu \mathrm{m}^{2}$ area, and the cytoplasm/nucleus ratio was $6.0 \pm 0.3$. The somata of most neurons were smooth, but some neurons contained numerous elevations and depressions of the soma surface (Figure 1).

\section{Hillock and axon}

Most of the neurons had the conical shaped axon hillock, the limits of which were hardly discernible (Figure 1). The axonal hillock of $9 \%$ 
neurons, however, was well discernible and covered $135 \pm 52 \mu \mathrm{m}^{2}$. The axon was a $2-6 \mu \mathrm{m}$ thick process originating from the hillock. We could trace the axon for up to $1.6 \mathrm{~mm}$. In $23 \%$ of neurons, the axon was bifurcated and had collaterals (Figure 1C). Most of the neurons were classified as Golgi I type neurons because they had a long prominent axon (Figure 1A-C). A few neurons (5\%) were classified as Golgi II type neurons, as their processes terminated in close proximity to the somata (Figure 1D).
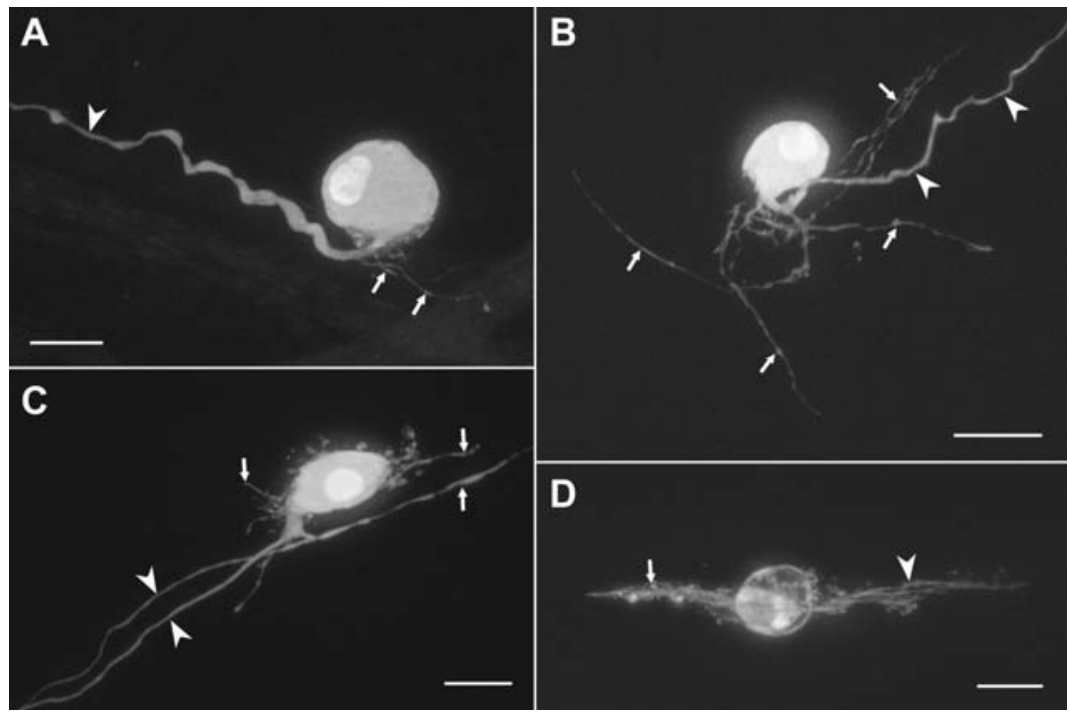

Figure 1. Frog intracardiac neurons labelled by the injection of Lucifer Yellow CH (A) and AlexaFluor 586 (B-D). Note the structural variability of the axon (arrowheads) and other processes (arrows). The processes of varying length originate from the axonal hillock, soma and proximal axon (A-C). Bubble shaped extensions of the soma surface are seen in C. Neurons in panels A-B are classified as the unipolar while in C-D as bipolar. The neuron in panel D is classified as of the Golgi II type, because all its processes terminate in close proximity to the soma. $\mathrm{Bar}=25 \mu \mathrm{m}$. 


\section{Neuronal processes}

Multiple neuronal processes originated from the soma, hillock and the proximal axon (Figures 1,2). With respect to the long axis of the soma, the neuron contained $3.5 \pm 0.3$ long and $5.5 \pm 0.6$ short processes. The initial segment of the axon had $4.3 \pm 0.6$ processes. The hillock and soma had $1.7 \pm 0.5$ and $4.1 \pm 0.6$ processes, respectively. The average length of long processes was $86 \pm 5 \mu \mathrm{m}$ with the longest processes reaching up to $500 \mu \mathrm{m}$. The somata of $48 \%$ of the neurons had spine, bubble or flake like extensions (Figure 1C). Based on the position of the processes on the soma, $61 \%$ of neurons were classified as unipolar, $25 \%$ multipolar, 11\% bipolar and 4\% pseudounipolar (Figure 1).

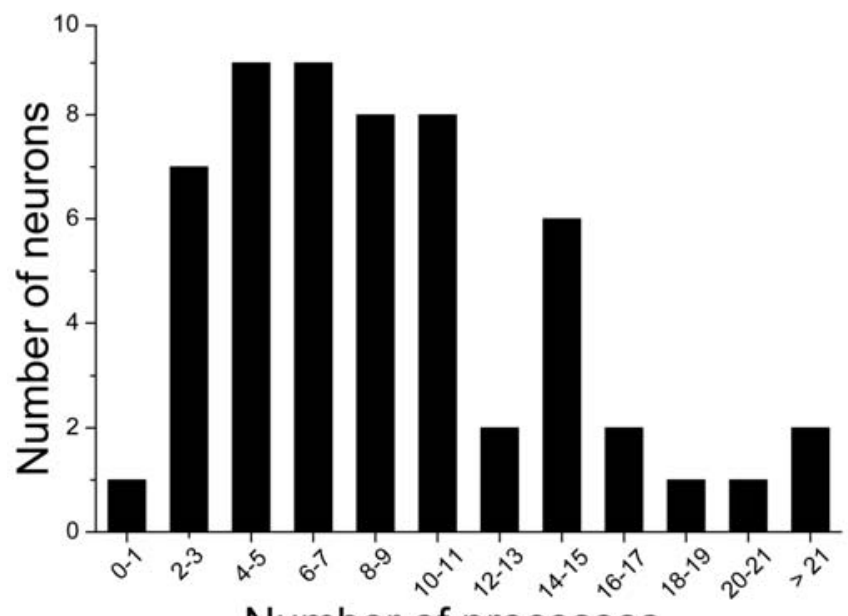

Number of processes

Figure 2. Number of processes among the labelled frog intracardiac neurons $(\mathrm{n}=56)$.

\section{DISCUSSION}

Our study demonstrates that the frog intracardiac neurons contain multiple processes which originate from the soma, hillock and the proximal axon. A small proportion of the frog intracardiac neurons is devoid of long processes. The frog intracardiac neurons contain fine extensions of the soma, and differ in morphology of the soma, the 
number and the length of processes. Both the Golgi I and II type neurons are present in the frog heart. Hence, we postulate that the frog intracardiac neurons are structurally more complex and diverse than it has been earlier considered.

Our findings contradict to a view that the frog intracardiac ganglia contain only adendritic neurons of the unipolar type [1,8]. The diverse morphology of intracardiac neurons has been reported in mammals including rat, guinea pig and dog [3-6]. In addition, the diverse morphology of sympathetic neurons has been shown in frog [9-10]. It was proposed that the different morphology of the intracardiac neurons reflects their distinct functional specializations [4].

In respect to the functional model of the frog intracardiac ganglion, all the intrinsic neurons of this ganglion serve the parasympathetic function $[1,8]$. The intracardiac neuron is innervated by $1-2$ presynaptic axons [1]. The terminals of presynaptic axons contact and innervate the initial segment of the postsynaptic axon [1]. The short processes of the soma identified in the present study probably represent the dendrites and may be considered as the sites of synapses with the presynaptic axon terminals. The processes that originate from the hillock and the proximal axon could serve as the local axon collaterals. Since these local axon collaterals contact the adjacent neuronal somata, they may exert the modulation effect on the neighbouring intracardiac neurons [9]. These findings we obtained and the discovery of the Golgi II type neurons suggest the morphological basis for communication between the frog intracardiac neurons. It is likely that the frog intracardiac ganglia are not a simple relay for preganglionic fibers, but could serve as a more complex integration centers.

The intracellular injection technique has been used in our study. This technique allows us to trace the axons and dendrites of the individual nerve cells. Although more work should be done in combining the intracellular injection with both the electron microscopy and immunocytochemistry, the presented data would contribute towards an understanding of the structure of the frog intracardiac ganglion. 


\section{REFERENCES}

1. Gibbins I.L., Morris J.L. (2006) Structure of peripheral synapses: autonomic ganglia. Cell Tissue Res, 326, 205-220.

2. Pannese E. (1994) Different types of neuron. In: Neurocytology. Thieme Medical Publishers. New York. 15-23.

3. Xi X.H., Thomas J.X., Randall W.C., Wurster, R.D. (1991) Intracellular recordings from canine intracardiac ganglion cells. J Auton Nerv Syst, $32,177-182$.

4. Edwards F.R., Hirst G.D., Klemm M.F., Steele P.A. (1995) Different types of ganglion cell in the cardiac plexus of guinea-pigs. J Physiol, 486 (Pt 2), 453-471.

5. Richardson R.J., Grkovic I., Anderson, C.R. (2003) Immunohistochemical analysis of intracardiac ganglia of the rat heart. Cell Tissue Res, 314, 337-350.

6. Pauza D.H., Skripkiene G., Skripka V., Pauziene N., Stropus R. (1997) Morphological study of neurons in the nerve plexus on heart base of rats and guinea pigs. J Auton Nerv Syst, 62, 1-12.

7. McMahan U.J., Kuffler S.W. (1971) Visual identification of synaptic boutons on living ganglion cells and of varicosities in postganglionic axons in the heart of the frog. Proc R Soc Lond B Biol Sci, 177, 485508 .

8. Heathcote R.D., Sargent P.B. (1987) Growth and morphogenesis of an autonomic ganglion. I. Matching neurons with target. J Neurosci, 7, 2493-2501.

9. Forehand C.J., Konopka L.M. (1989) Frog sympathetic ganglion cells have local axon collaterals. J Comp Neurol, 289, 294-303.

10. Feruzzi D., Forehand C.J. (1994) Morphology of two classes of targetspecific bullfrog sympathetic preganglionic neurons. J Comp Neurol, $341,315-323$.

\section{Address for correspondence:}

Darius Batulevicius

Lithuanian University of Health Sciences

Institute of Anatomy, Medical Academy

A. Mickeviciaus Street 9, LT-44307 Kaunas, Lithuania

E-mail: batuda@med.kmu.lt 